\title{
Design and Experimental Investigations of Magentic Energy Harvester at Low Resonance Frequency
}

\author{
Ala'aldeen Al-Halhouli ${ }^{\mathrm{a},{ }^{c} *}$, Hussam A. Kloub ${ }^{\mathrm{b}}$, Michael Wegner ${ }^{\mathrm{c}}$, Stephanus Büttgenbach ${ }^{\mathrm{c}}$ \\ ${ }^{a}$ Mechatronics Engineering Department, German Jordanian University, Amman, Jordan \\ ${ }^{b}$ Department of Biomedical Engineering, German Jordanian University, Amman, Jordan \\ ${ }^{c}$ Institute of Microtechnology, Technische Universität Braunschweig, Germany
}

\begin{abstract}
Energy harvesting replaces the traditional method of using batteries to power electronic devices and sensors. It makes them self-sufficient, autonomous, and long-living and requires lower maintenance costs. This paper presents a vibration based electromagnetic energy harvester. The experimental work in this paper investigates the harvester design parameters: the magnet position, the number and the size of magnets and the geometry of the coils. Experimental results show that the optimized prototype generates $9 \mathrm{~mW}$ at 120 load resistance. This energy would be sufficient for energy supply of sensors and electronic devices such as mp3 players or mobile phones.
\end{abstract}

Keywords: energy harvesting, vibration energy, electromagnetic, micro generator

\section{Introduction}

Energy harvesters or micro energy generators are an approach proposed to replace batteries in sensors and low power electronic systems in order to realize autonomous electronic applications. Such autonomous systems reduce the maintenance costs, enhance the mobility, and prolong the life-time of the device [1-4].

Harvested energy can be in the form of thermal, solar, vibration or electromagnetic energy. Operation concepts for energy harvesting devices are based on piezoelectric, capacitive, magnetic, photo voltaic or thermoelectric concepts [1-8].

Selection of an operation concept relies mainly on the target application. Vibration energy is a very attractive source for developing related harvesters. It can be observed in various applications such as motors, automobiles, home electric appliances such as wash machines and refrigerators, and even the human motion generates vibration energy [1-4]. Such applications are potential sources for driving associate electronic systems by utilizing vibration energy harvesting. However, the frequencies of such vibrations are low, i.e. in the range of 2 to $300 \mathrm{~Hz}$.

State-of-the-art harvesters of ambient vibration energy, namely piezoelectric and electrostatic harvesters exhibit still very small efficiency. However, magnetic based harvesters show promising results compared to other concepts [3].

In this paper, a low frequency vibration energy harvester based on magnetic conversion is investigated. While state-of-the-art magnetic harvesters are based on massspring resonator systems, the proposed magnetic harvester is a free standing mass resonator. No mechanical spring is used. Such a design should enable the flexibility to operate the device at different frequencies, without limitation to resonance frequencies as in the case of mass-spring resonators.

${ }^{*}$ Corresponding author. Tel.: +962- 6-429 4511

e-mail: alaaldeen.alhalhoul@gjuedu.jo

(C) 2015 International Association for Sharing Knowledge and Sustainability

DOI: $10.5383 /$ ijtee.10.01.011 


\section{Concept of Operation}

The design of the magnetic based vibration energy harvester is shown in Fig. 1. The device consists of a movable and a fixed magnet, enclosed in a frame. The frame is surrounded by a conductor coil mounted between two mechanical stoppers.

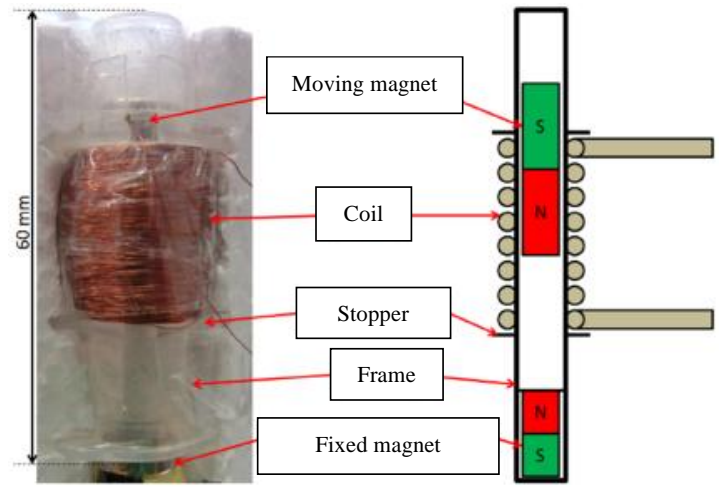

Fig.1: Design of magnetic based vibration energy harvester.

The movable and the fixed magnet are installed such that similar magnetic polarity sides are opposed to each other. This arrangement causes magnetic field repelling. Therefore the movable magnet is displaced at a specific distance away from the fixed magnet.

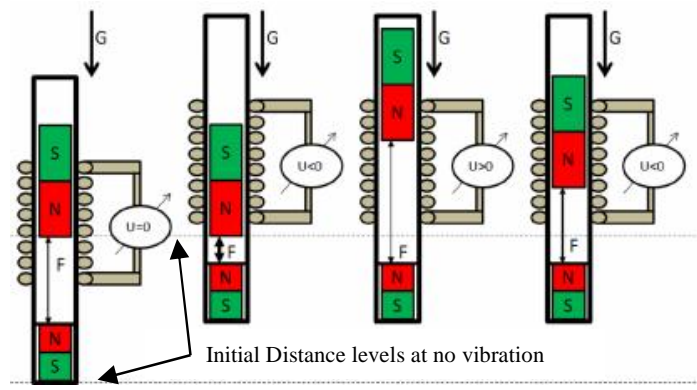

Fig.2: Mechanical excitation response of magnetic based vibration energy harvester.

The schematic diagram of mechanical excitation response of the magnetic based vibration energy harvester is shown in Fig. 2.

Initially, at no external excitation, the movable magnet is at a specific position. The externally induced vibration of the device causes the movable magnet (movable mass) to vibrate within the frame. Consequently, the magnetic field of the movable mass crosses the outer coil at different positions and induces a varying magnetic field flux in the coil. According to Faraday's law, this varying magnetic flux induces an electrical current in the coil.

When the external mechanical excitation stops, the movable mass continues to vibrate for a specific time period before settling down. This response is due to the interaction between the movable and the fixed magnetic fluxes.

\section{Design \& Experiment Set Up}

The magnetic harvester device consists mainly of a container, two magnets, and a coil. The container is a cylinder with diameter of $12 \mathrm{~mm}$. The cylindrical magnets are made of nickel coated NdFeB (M1D08-054N38). The movable magnet has a height of $15 \mathrm{~mm}$ and diameter of $12 \mathrm{~mm}$. The fixed magnet has a height is $5 \mathrm{~mm}$ and a diameter of $12 \mathrm{~mm}$. The coil has a length of $24 \mathrm{~mm}$, a diameter of $23.7 \mathrm{~mm}$, and 1500 number of turns. The rod diameter is $0.2 \mathrm{~mm}$.

The experimental setup consists of a function generator, an amplifier (LM1876), a loudspeaker, the magnetic harvester, a USB data acquisition card (NI USB 6259), and a PC station with Labview software interface.

The function generator is used to generate a sinusoidal signal with very low frequency. This signal is the basic excitation signal. The output signal used in the experiment is $50 \mathrm{mV}$ at $3.7 \mathrm{~Hz}$ and at $90 \mathrm{~Hz}$.

The output of the function generator is directly connected to the amplifier which generates an output signal of $20 \mathrm{~W}$. The amplifier is supplied with a DC voltage of $30 \mathrm{~V}$. The output of the amplifier is connected to the loudspeaker in order to generate mechanical vibration, whereby the vibration frequency and amplitude correspond to the electrical frequency and voltage signal of the function generator.

The magnetic energy harvester is mounted on the loudspeaker in such a way that the mechanical vibration of the loudspeaker excites the harvester mechanically. The output connectors of the harvester coil are fed to a resistance load.

The induced voltage signal of the harvester is measured and transferred to a PC station by a USB data acquisition card. The transferred data is stored and displayed based on a Labview program.

\section{Experimental Results}

In first experiments the optimal loading resistance has been measured. The load resistance is changed and the resultant output voltage is measured. Then, the output power is calculated from the measured induced voltage. The optimal load response is shown in Fig.3.

The measured induced power at a frequency of $3.7 \mathrm{~Hz}$ is less than the measured induced power at a frequency of $90 \mathrm{~Hz}$. However, for both frequencies, the optimal load resistance is $120 \mathrm{Ohm}$.

The voltage response of the harvester at a frequency of $90 \mathrm{~Hz}$ and $120 \mathrm{Ohm}$ load resistance is shown in Fig.4.

The measured optimal power is $1.4 \mathrm{~mW}$, resulting from a semi-sinusoidal waveform signal, with positive peak of $1.4 \mathrm{~V}$ and negative peak of $-1.5 \mathrm{~V}$. 


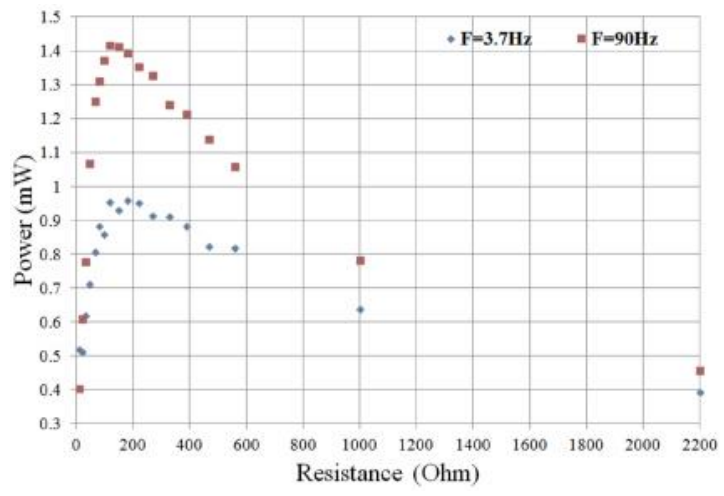

Fig.3: Optimal load response.

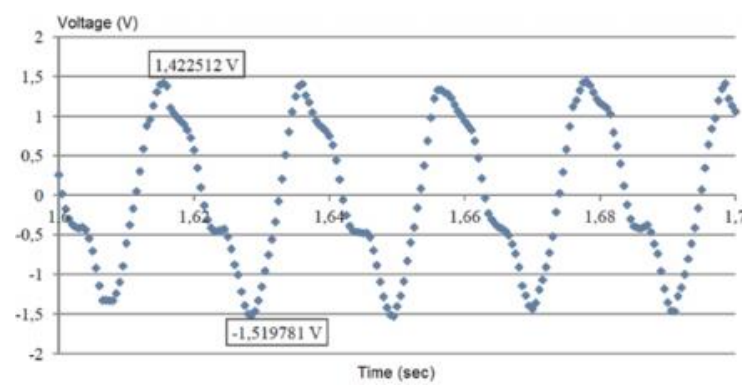

Fig.4: Voltage response at optimal load.

The next measurements have been performed for four prototypes with different initial movable magnet displacement. These initial displacements are set by changing the location of the fixed magnet, so that its magnetic field affects the location of the movable magnet.

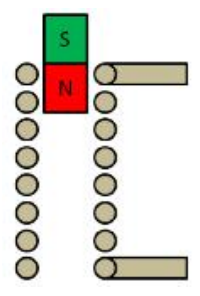

Prototype 1

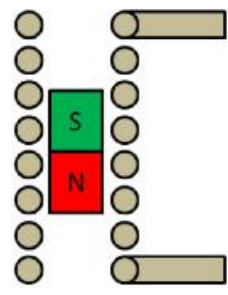

Prototype 3

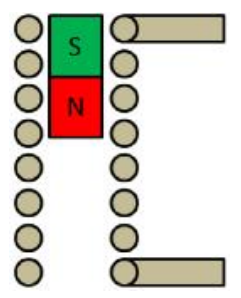

Prototype 2

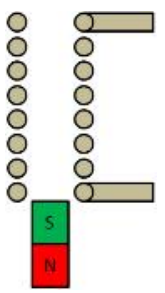

Prototype 4
Fig.5: Design optimization of movable magnet.

The first prototype (P1) is designed such that the middle of the magnet overlaps with the upper section of the coil, while the remaining part of the magnet is outside the coil. The second prototype (P2) is designed with complete overlap between the magnet and the upper section of the coil. In the third prototype (P3) the complete magnet is located in the middle of the coil. In the fourth prototype $(\mathrm{P} 4)$ the magnet is completely outside the coil.

The measurements of the induced power are shown in Fig.6. The first prototype shows clearly the largest induced power $(1.5 \mathrm{~mW})$ compared to the other designs. Interestingly, the optimal power values for different prototypes were observed at different excitation frequencies.

The third and fourth prototypes almost have the same optimal frequency, which is around $55 \mathrm{~Hz}$. The second prototype has an optimal frequency of $30 \mathrm{~Hz}$. On the other hand, the first prototype, with the largest induced power value, shows optimal frequency at $91.5 \mathrm{~Hz}$, which differs significantly from the others.

The next measurements have been performed for prototypes with different coil lengths/diameter: $15.8 \mathrm{~mm} / 25.5 \mathrm{~mm} \quad(\mathrm{P} 1), \quad 17.3 \mathrm{~mm} / 21 \mathrm{~mm} \quad(\mathrm{P} 2)$, $20 \mathrm{~mm} / 26 \mathrm{~mm}(\mathrm{P} 3)$, and $21.3 \mathrm{~mm} / 19 \mathrm{~mm}(\mathrm{P} 4)$. However, for all prototypes, the number of turns is 1000 . The power responses of the new prototypes are shown in Fig.7.

The order of prototypes in Fig. 7 commencing with the largest induced power values is $\mathrm{P} 4, \mathrm{P} 1, \mathrm{P} 2$, and $\mathrm{P} 3$. All prototypes have optimal frequencies close to each other between $44 \mathrm{~Hz}$ to $48 \mathrm{~Hz}$. The measurements show that the shorter coil is corresponding with the largest power, except the case of $\mathrm{P} 4$, where largest power was observed due to the significant smaller coil diameter, that becomes much closed to the movable magnet, closer to magnetic field, thus more power transduction.

The last measurement investigates different impedance loads: a load resistance of $120 \mathrm{Ohm}$ in parallel with capacitors of different capacitance values of $470 \mathrm{nF}$, $22 \mu \mathrm{F}, 100 \mu \mathrm{F}, 470 \mu \mathrm{F}$, and $2200 \mu \mathrm{F}$. The voltage response of the different impedance loads is shown in Fig. 8.

The measurements show that the higher the capacitance value, the higher is the mean value of the induced voltage.

\section{Design optimization}

The size of energy harvester is a crucial design parameter for flexible integration of harvester in an electronic application. The harvester size miniaturization and output power generation is a trade-off. However, the topology of the coil design; circular, rectangular, planar, or vertical should be firstly investigated before the design optimization.

Micromachining of three dimensional (3D) devices is more complicated than the planar ones; therefore for harvester size miniaturization the planar coil topology is more suited than the vertical one. 
The following is design evaluation of circular and rectangular coils. The first evaluation in Fig. 9 is for inductance vs. wire diameter of the coil from previous study [4]. Obviously, circular wire has larger inductance than the rectangular or square wire. For both topologies, inductance decreases as the wire diameter is increasing. But at relatively very large diameter, both circular and square wires are nearly having the same inductance.

The following evaluation in Fig. 10 is for resistance vs. wire diameter of the coil from previous study [4]. In this case, the circular coil has larger resistance than the square one. Also, at relatively larger wire diameter, both circular and square coil have almost identical resistance.
From above evaluations, the circular coil should be selected for design optimization. In order to experimentally verify this conclusion, three different micro coil designs are tested. One square coil design (wq-15) with diameter $15 \mu \mathrm{m}$, and two circular coil designs; (w-10) diameter is $10 \mu \mathrm{m}$ and (w-15) diameter is $15 \mu \mathrm{m}$. The coils are mounted on a permanent magnet and electrical current is passed through the coils. The passing current through the coils induces magnetic field, appears as resultant magnet deflection, which is measured by force sensor. The responses of the tested coils are shown in Fig. 11.

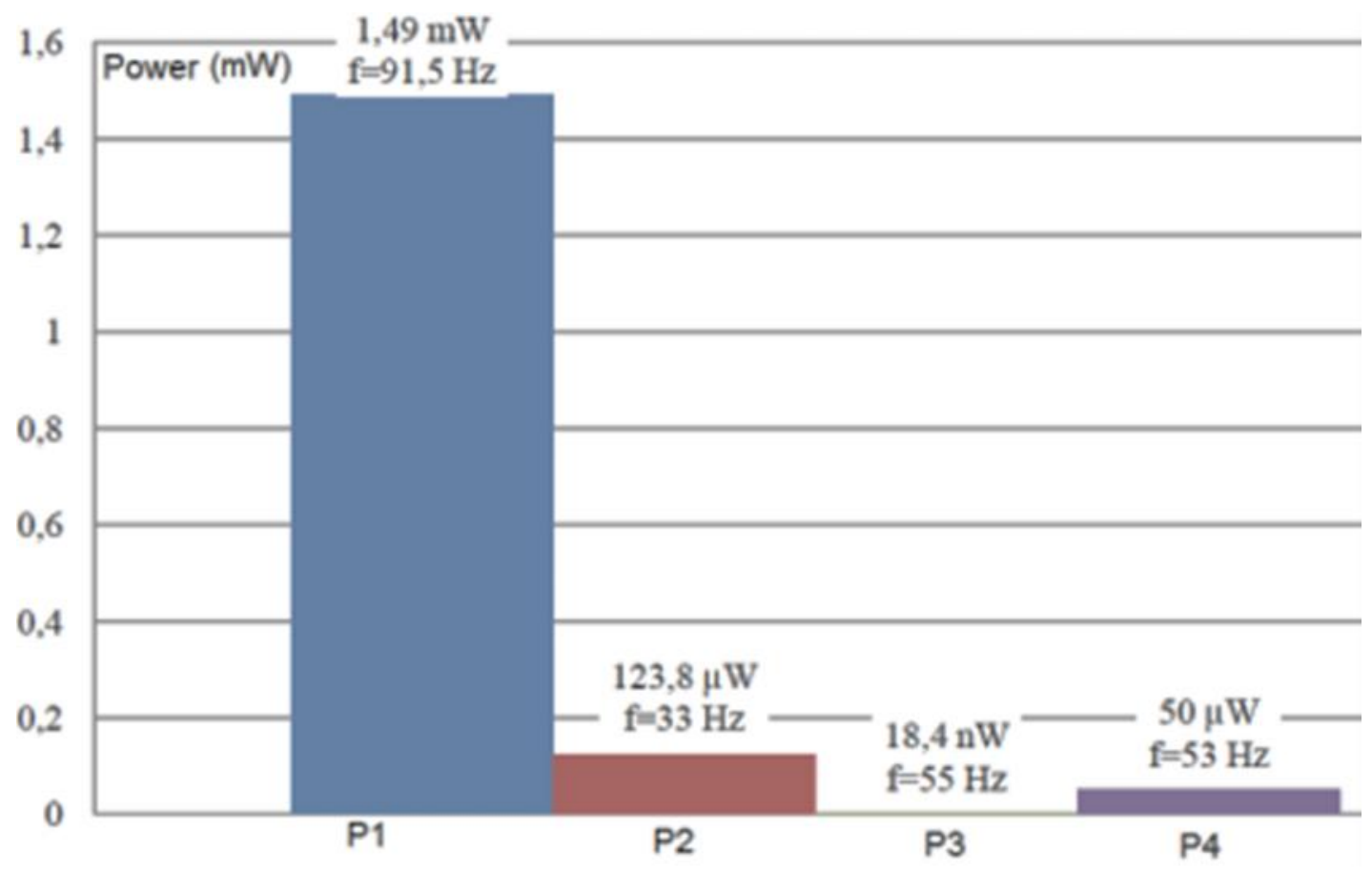

Fig.6: Optimal power measurements for different prototypes. 


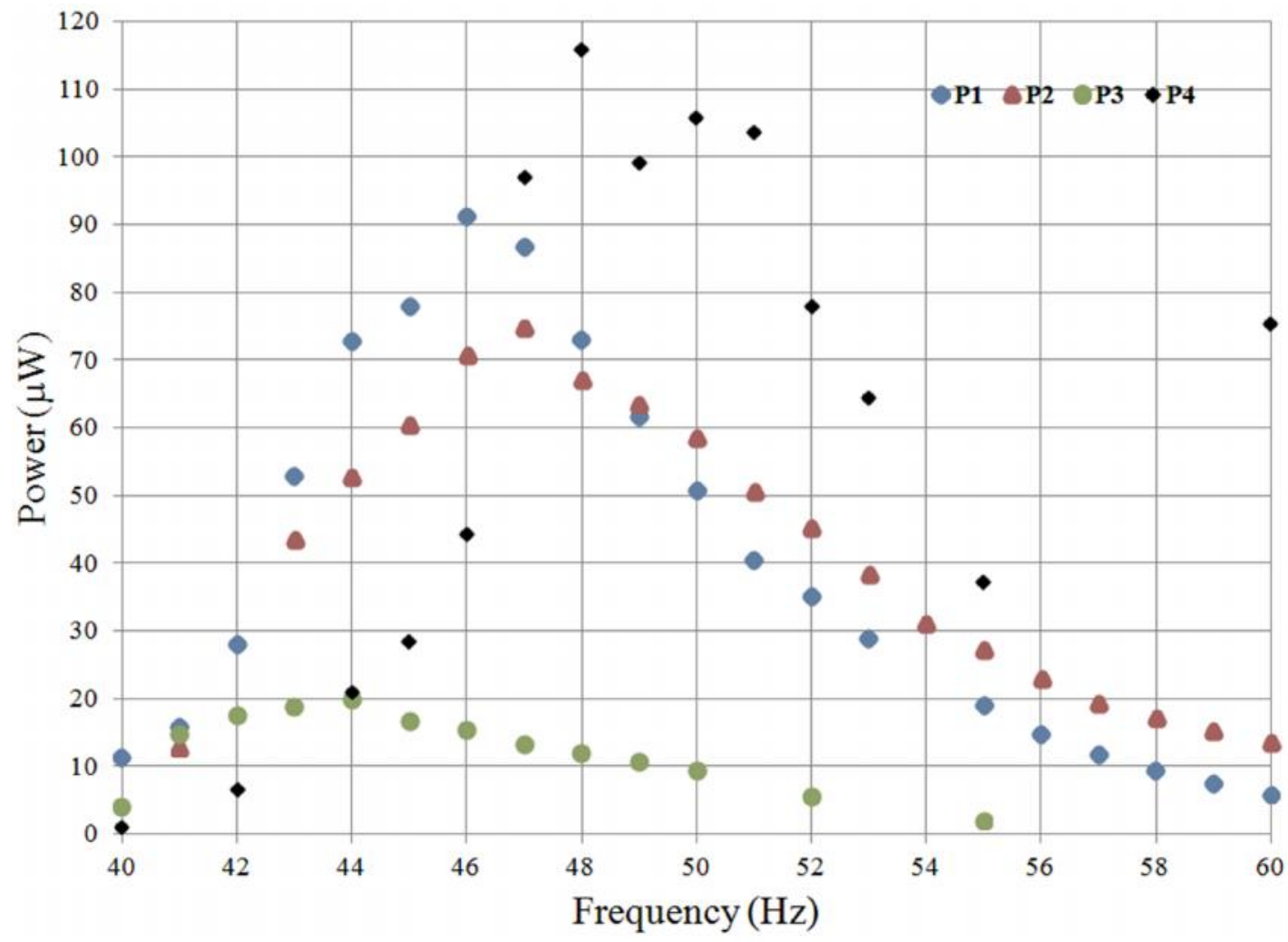

Fig.7: Optimal power measurement for different coils design prototypes.

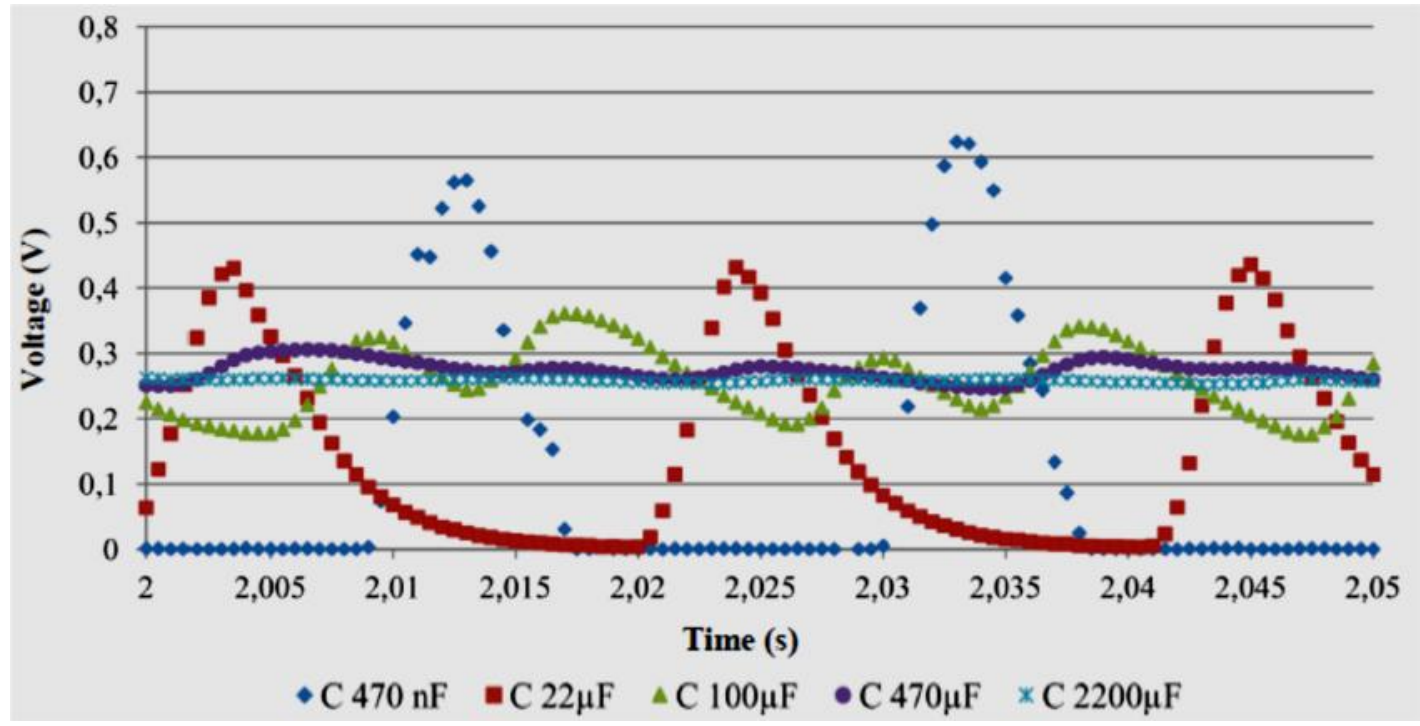

Fig. 8: Voltage response at different impedance loads. 


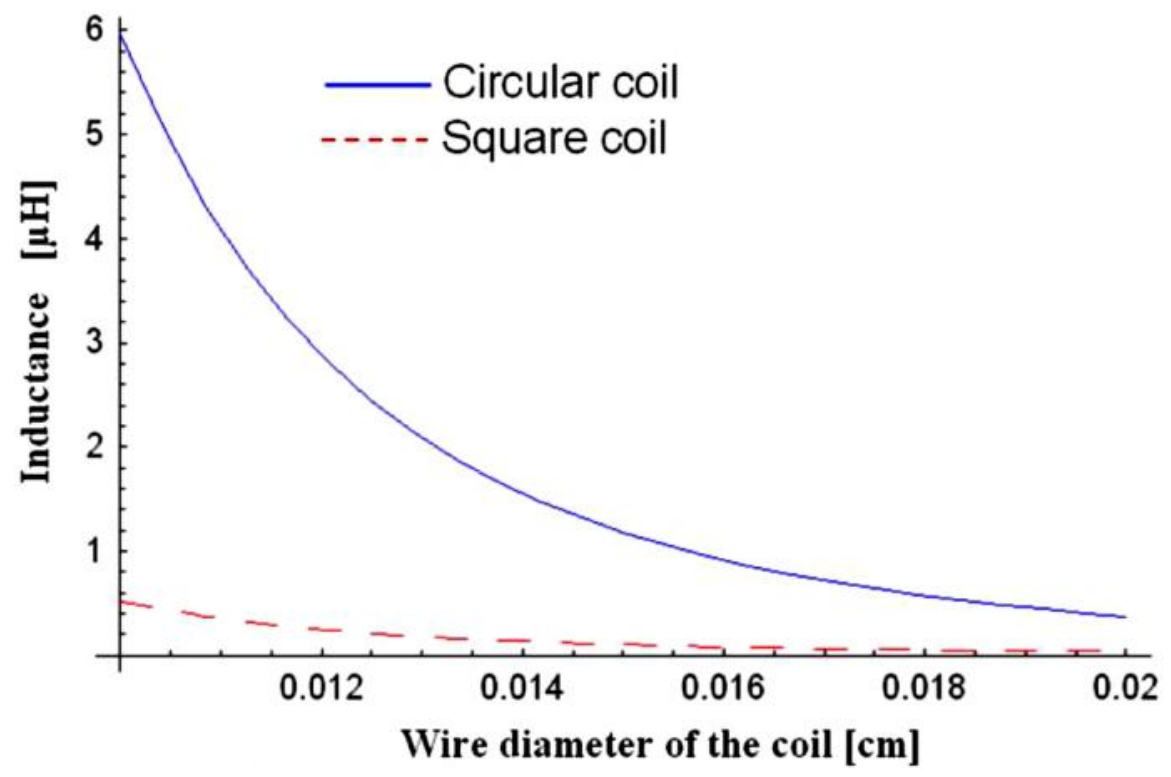

Fig. 9: Inductance response.

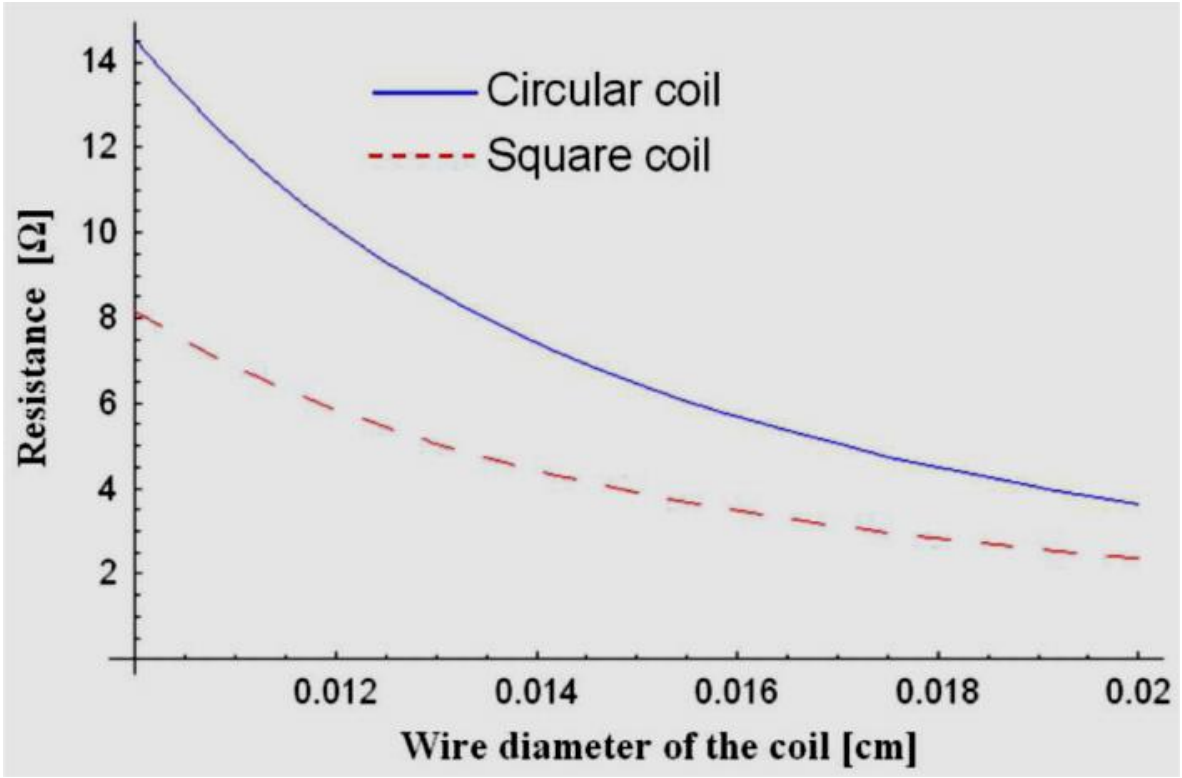

Fig. 10: Resistance response. 


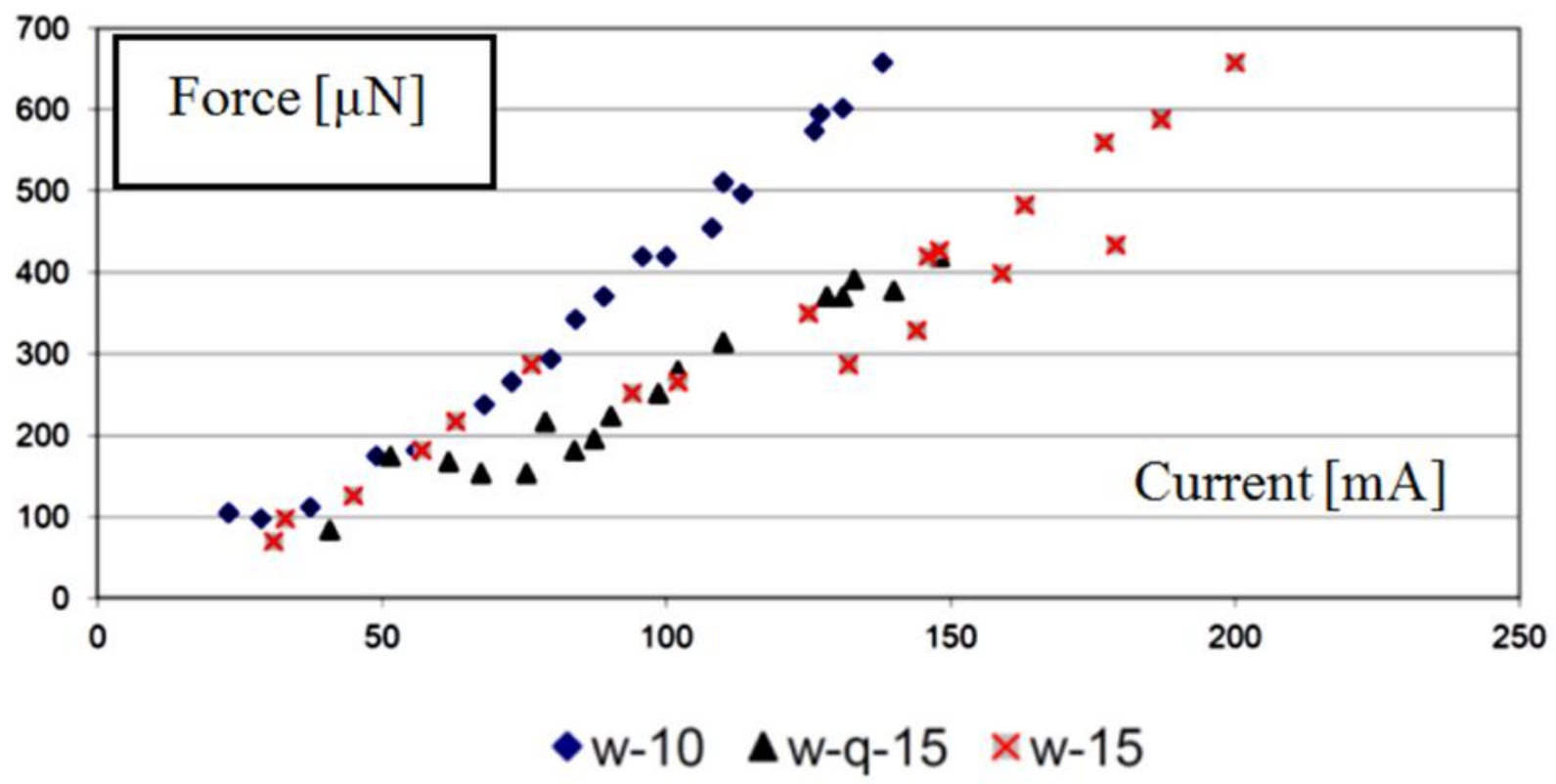

Fig. 11: Force response.

The square coil shows lower force response than circular wire at lower input current values. On the other hand, circular wire with smaller diameter; $10 \mu \mathrm{m}$ has the largest force response among others. This experimental result verifies that circular wire with smallest diameter results in largest induced magnetic field.

An important design parameter is the positioning of the coil. In general, permanent magnet is located to observe parallel movement with respect to the planar coil. Knowing the directions of magnetic field and magnet deflection, then the direction of the resulting voltage is easily observed. Therefore, the position of the coil plays important role in design optimization as it is described below.

The following is analysis of three different positioning topologies of planar coils. The first topology in Fig.12 shows the planar coil completely adjusted to be in the center of the permanent magnet, such that the permanent magnet movement is parallel to the coil. The arrows in figure are pointing at the direction of the induced voltage in the coil. Obviously, the lateral parts of the coil have minimum induced voltage components compared with the vertical parts. Thus, the coil design is modified into the form shown in Fig. 13.

The second topology in Fig. 13 shows conductor bars instead of a coil. The bars are fixed such that the movement of permanent magnet is parallel to the bars. The arrows are pointing at the direction of the induced voltage in the conductors. In order to best utilize the induced electrical energy, all conductor bars are connected together as shown in Fig. 14; topology 3.

The third topology has lateral bars which results in similar problematic as in topology 1 . Also, by such lateral bars, the induced electrical current increases while the corresponding induced voltage is decreased, and this will result in more energy loss due to conductor resistance.

An approach to overcome the problem of design topology 3 is by designing multi-layer planar coil as shown in Fig. 15. Each layer consists of three conductor bars such that the induced voltage value increases as the bar distance from permanent magnet is increased. The bars with smallest induced voltage are used for electrical connection with other layers. 


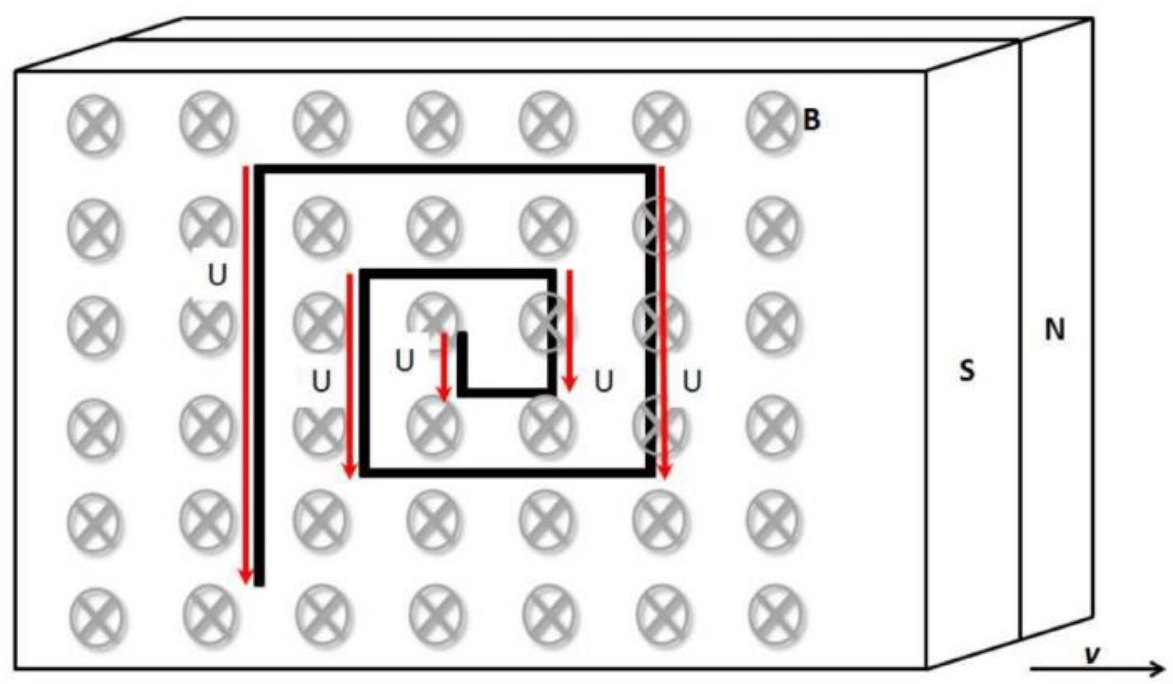

Fig. 12: Coil positioning-topology 1.

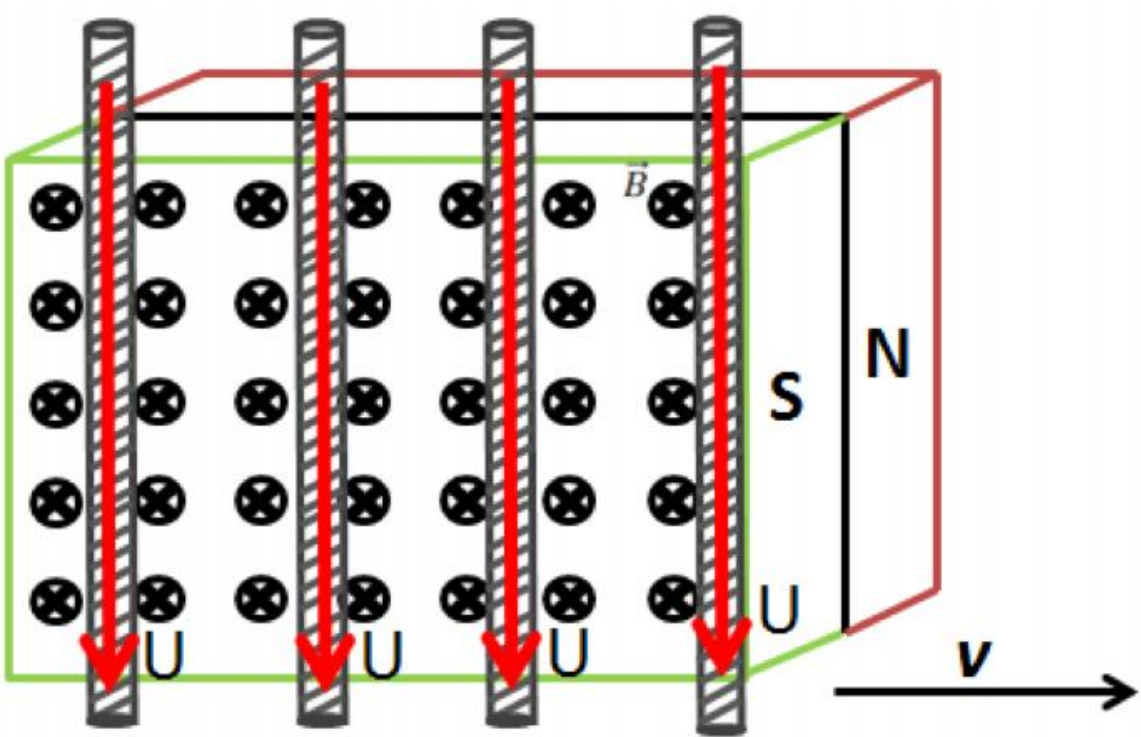

Fig. 13: Coil positioning-topology 2. 


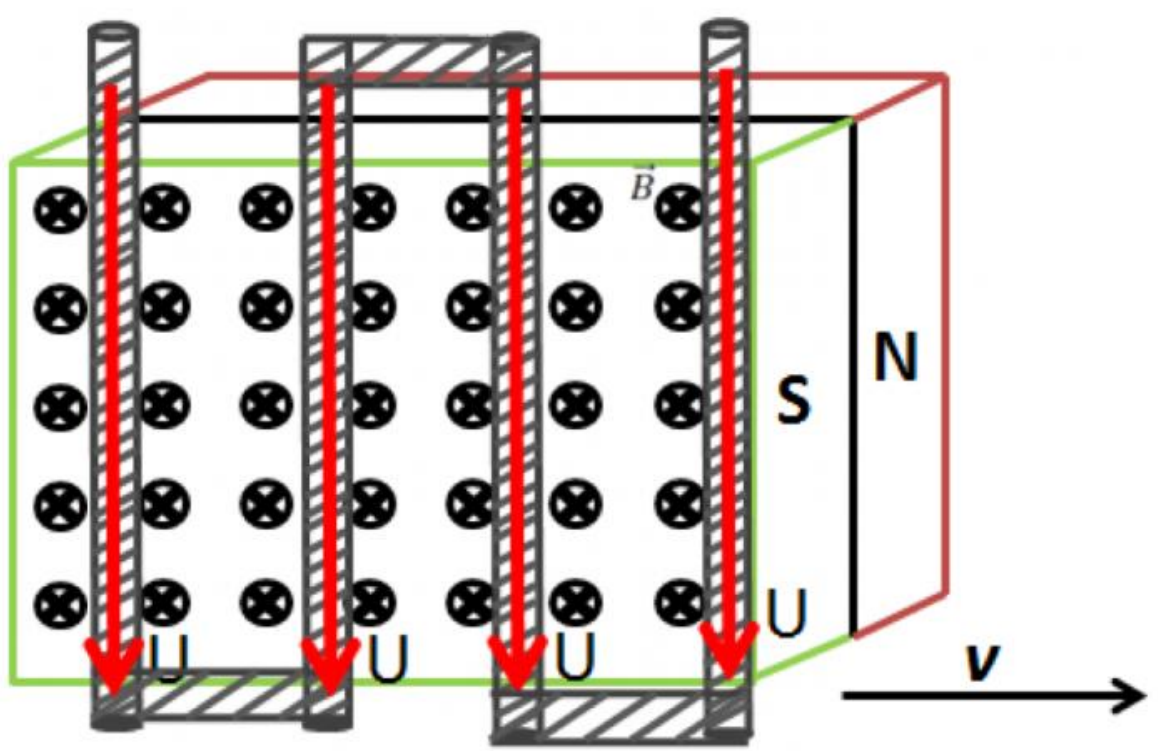

Fig. 14: Coil positioning-topology 3.

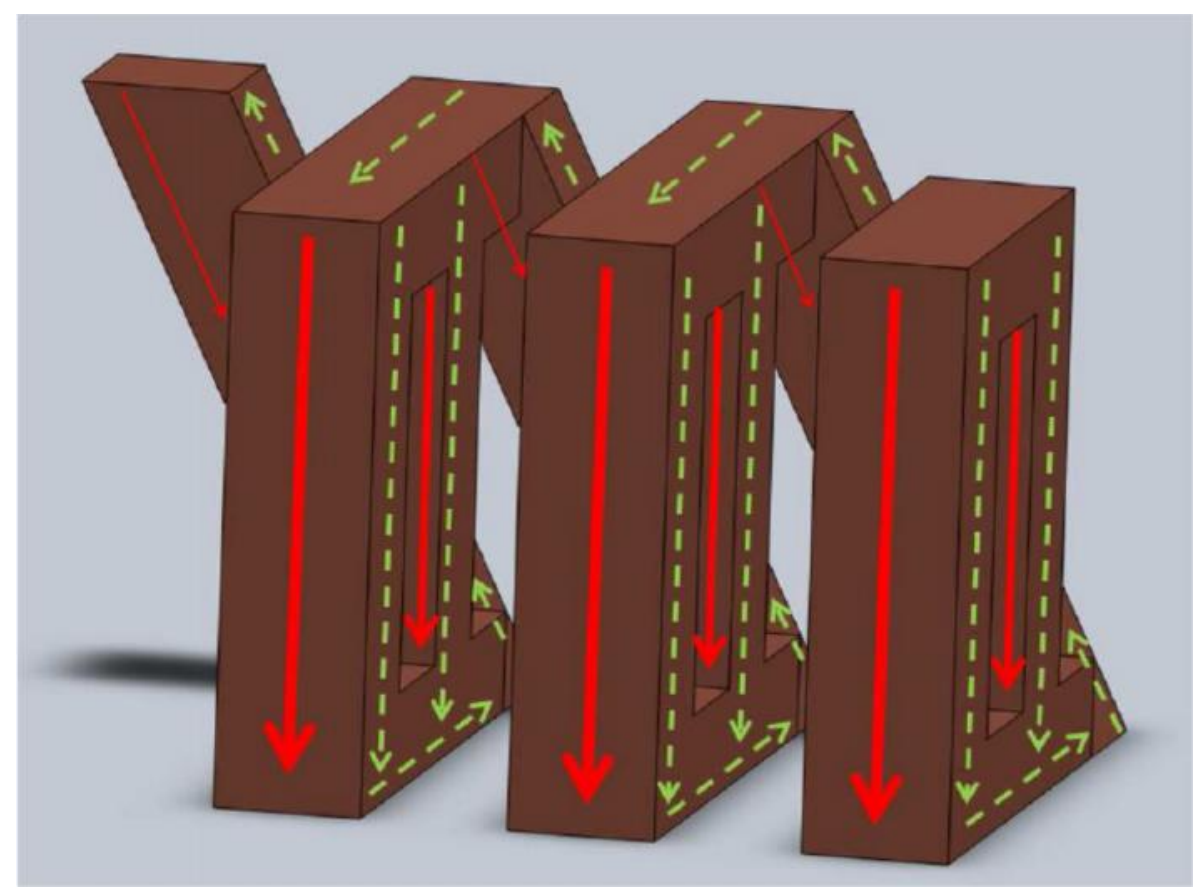

Fig. 15: Optimized coil design. 


\section{Conclusion}

A free standing mass resonator harvester based on magnetic conversion has been proposed in this paper. Measurements of different prototypes have been performed in order to investigate the induced power response and to optimize the harvester design.

The optimal design features maximum coil length and half overlap between the movable magnet and the upper section of the coil.

Design optimization of the coil requires planar design, with multi-layer such that each layer consists of many conductor bars connected together. This approach is for keeping larger value of induced voltage in the coils.

\section{Acknowledgments}

One of the authors (S.B.) thanks the Volkswagen Foundation for financial support.

\section{References}

[1] Wegner M, Al-Halhouli A T. Elektromagnetischer Energiewandler im niedrigen Frequenzbereich, Institute für Mikrotechnik, 2011.

[2] Kloub H. High efficient micro electromechanical capacitive transducer for kinetic energy harvesting,
Ph.D. Dissertation, Albert Ludwigs University Freiburg, 2011.

[3] Emmanuel B. Printed circuit board-based electromagnetic vibration energy harvester, Ph.D. Dissertation, Albert Ludwigs University Freiburg, 2010.

[4] Saha C R, O'Donnell T, Wang N, McCloskey Electromagnetic generator for harvesting energy from human motion, Sensors and Actuators-A: Physical, 2008, 147: 248-253.

[5] Romero E, Neuman M R, and Warrington R O, Kinetic energy harvester for body motion (WIMS) Michigan Technological University, Houghton, USA, 2009, pp. 237-240.

[6] Krähenbühl D, Zwyssig C, Weser H and Kolar J. Mesoscale Electric Power Generation From Pressurized Gas Flow Power Electronic Systems Laboratory, J. Micromech. Microeng. 2009; 19 (9): 094009.

[7] Ahmed I, Majid Butt M, Psomas C, Mohamed A, Krikidis I, Guizani I. Survey on energy harvesting wireless communications: Challenges and opportunities for radio resource allocation, Computer Networks, 2015, 88: 234-248

[8] Chena R, Rena L, Xiaa H, Yuana X, Liub X. Energy harvesting performance of a dandelion-like multidirectional piezoelectric vibration energy harvester, Sensors and Actuators A: Physical, 2015, 230: 1-8 